\title{
MODULATED STRUCTURES IN THE CONDENSED MATTERS WITHOUT LIFSHITZ INVARIANT
}

\author{
A. I. Olemskoi ${ }^{\dagger}$, S. V. Berezovsky ${ }^{\dagger}$, V. F. Klepikov ${ }^{\dagger \dagger}$, Yu. V. Sereda ${ }^{\dagger \dagger}$ \\ ${ }^{\dagger}$ Sumy State University, 2 Rymskyi-Korsakov Str., UA-244007 Sumy, Ukraine \\ ${ }^{\dagger \dagger}$ Scientific and Technological Center of Electrophysics, National Academy of Sciences of Ukraine \\ 28 Chernyshevskyi Str., P.O.BOX 8812, UA-310002 Kharkiv, Ukraine \\ tel.: +380-572-404-720, fax:+380-572-474-507, e-mail: ipct@pem.kharkov.ua
}

(Received April 20, 1998)

\begin{abstract}
The non-linear properties of order parameter distributions $\varphi(x)$ in the condensed matter without Lifshitz invariants are considered numerically. It is shown that the analytical approach developed in [1-4] describes quantitatively the parametric evolution of the order parameter distributions with the decrease of temperature. The metastable states and possible relaxational features of such a system are discussed.
\end{abstract}

Key words: Landau theory of phase transitions, order parameter, spatial distribution, Jacobi elliptic functions.

PACS number(s): 05.70.Fh, 64.60.My, 64.70.Kb

\section{INTRODUCTION}

The description of phase transitions in the systems with one-component order parameter is an example of theoretical approach which can be useful not only for the investigation of concrete condensed matters (e.g., sodium nitrite, thiourea, some magnets) but for the consideration of fundamental problems of the phase transition theory and self-organization phenomena, in particular, the appearance of stable space-nonhomogeneous structures, as well.

In papers [1-4] the new analytical approach has been developed in the framework of a model which generalizes the Landau theory of phase transitions. This approach allows to find the novel approximate solutions for the spatial distributions of one-component order parameter in the systems with competitive interactions. The advantage of this approach is, in particular, that it gives an algorithm of constructing essentially non-linear successive approximations to the exact solution of the variational equation. In the simplest case these solutions can be expressed through the elliptic Jacobi functions. These functions are more convenient in the analysis and permit to get more information about the properties of incommensurate distributions of order parameter than the usually used approximations with the Fourier series which, in general, can be infinite $[5,6]$.

However, the variational equation for the considered problem is a non-linear differential equation the exact analytical solutions of which is not known in general. This circumstance is the reason why the numerical methods are used for solving this variational problem [5].

In the present paper, we numerically investigate the properties of equilibrium distributions of one-component order parameter (OP) in the incommensurate phase. Such a consideration is important to evaluate the precision of the approximate analytical distributions found in [1-4] and for the detailed clarification of their physical meaning. Moreover, the obtaining of information about the structure of thermodynamical potential (TP) in the vicinity of its absolute minimum is an useful preliminary stage of the investigation of relaxational properties of the considered system.

\section{THE NUMERICAL SOLUTION OF VARIATIONAL EQUATION}

The incommensurate states of one-component OP can be described with the help of the following TP [1-4]:

$\Phi=\int_{0}^{L}\left[\left(\varphi^{\prime \prime}\right)^{2}-g\left(\varphi \varphi^{\prime}\right)^{2}-\gamma\left(\varphi^{\prime}\right)^{2}+q \varphi^{2}+\frac{p}{2} \varphi^{4}+\frac{1}{3} \varphi^{6}\right] d x$

where $\varphi(x)$ is the order parameter; $g, \gamma, q, p$ are the reduced material parameters; $\varphi^{\prime}$ stands for the spatial derivative; $L$ is the crystal length along the modulation axis. The phase transitions are assumed to be caused by the change of temperature $T$. The effective temperature $q$ has the form $q=q_{0} \cdot\left(T-T_{0}\right)$, where $q_{0}, T_{0}$ are some constants. Note that all variables in formula (2.1) are dimensionless.

The variational equation for the functional (2.1) is the following non-linear differential equation of the forth order:

$$
\varphi^{(I V)}+g\left(\varphi^{2} \varphi^{\prime \prime}+\varphi \varphi^{2}\right)+\gamma \varphi^{\prime \prime}+q \varphi+p \varphi^{3}+\varphi^{5}=0
$$

The finite difference method (see, for example, [7]) or the method of truncated Fourier series [8,9] are usually used for the numerical solution of the variational 
equations which describes the OP incommensurate structures. In the latter case the OP periodic distribution is expressed through the harmonic series with a finite number of harmonics. The wave number $b$ and the expansion coefficients of such series are initially assumed to be unknown.

The application of these methods shows that presumably the finite difference method gives more reliable results. For example, for the systems with two component OP it predicts that for the different values of TP parameters the transitions from the incommensurate state into the commensurate phase could be either of the first order or of the second order [5,7]. At the same time the method of Fourier series predicts the transitions only of the second order regardless the values of TP parameters $[5,8]$ which is in contradiction with the existing experimental data $[6]$.

We find the OP equilibrium distributions for the variational problem (2.1) $-(2.2)$ in three stages. On the first stage the solution of equation (2.2) is found with the help of 4th order Runge-Kutta method [10] with some set of initial conditions $S_{0}=\left\{\varphi(0), \varphi^{\prime}(0), \varphi^{\prime \prime}(0), \varphi^{\prime \prime \prime}(0)\right\}$. Then the TP value (2.1) is calculated. On the last stage the TP absolute minimum is defined for all initial conditions $S_{0}$ used.

The investigation is carried out for different values of material parameters. The parameter $p$ is always equal to unity: $p=1$. That is not the essential restriction of generality in the case when the properties of incommensurate state are considered $[5,9,11]$. The parameter $g$ is assumed to be negative [5,9]. For the given value of $g$ the effective temperature $q$ changes from $q_{I}$ (the point where the disordered phase loses its stability and transforms into the incommensurate state) to the temperature when the commensurate state becomes energetically favourable [4].

For system (2.1) the OP distribution in the incommensurate phase must not depend on the direction of axis $O X$ of the OP spatial modulation and on the direction of axis $O Y$ along which the ordering appears. Moreover, the distributions which differ only by the value of the phase $x_{0}$ are physically equivalent because the TP is invariant in respect to the phase shift $x \rightarrow x+x_{0}[5,12]$. We also assume that the function $\varphi(x)$ is a smooth periodic one. The consequence of the mentioned properties is that there always exists such a coordinate framework in which solutions describing the OP distributions in the incommensurate state are even or odd functions of the space coordinate and, moreover, the translation $x \rightarrow x+P / 4$ ( $P$ is the modulation period) changes the function evenness to the opposite one.

Such "even-odd" symmetry [13] allows to set two out of four initial conditions equal to zero, i.e. we can consider only the sets $S_{01}=\left\{0.0, \varphi^{\prime}(0), 0.0, \varphi^{\prime \prime \prime}(0)\right\}$ or $S_{02}=\left\{\varphi(0), 0.0, \varphi^{\prime \prime}(0), 0.0\right\}$. The application of sets $S_{01}$ and $S_{02}$ must give physically equivalent states when the periodic distributions are investigated. However, the OP space-homogeneous distribution in the commensurate phase can be obtained only for the set $S_{02}$. The last circumstance makes the application of the set $S_{02}$ more favorable.

The potential (2.1) and the equation (2.2) are invariant in respect to the reflection $\varphi \rightarrow-\varphi$. It means that if $\varphi(x)$ is the solution of equation (2.2) then $-\varphi(x)$ is the solution of (2.2) as well. This property also allows to make the volume of $S_{0}$-space twice as little.

\section{RESULTS AND DISCUSSION}

Some results obtained with the help of the considered numerical procedure are shown in the table. In this table the TP values calculated in the one-harmonic approximation $[9]$

$$
\varphi(x)=a \cdot \sin \left[b\left(x+x_{0}\right)\right]
$$

and for the distribution [1-4]

$$
\varphi(x)=a \cdot \operatorname{sn}\left[b\left(x+x_{0}\right), k\right]
$$

$(s n(x, k)$ is the elliptic Jacobi sinus) are also presented.

It follows from the table that model (3.4) is a good approximation for the description of OP equilibrium distributions in the incommensurate phase, in particular, in the vicinity of the point of transition into the commensurate state. It should be especially noted that the equilibrium value of $\varphi^{\prime \prime}(0)$ predicted by model (3.4) is closer to the numerical values of $\varphi^{\prime \prime}(0)$ then the $\varphi^{\prime \prime}(0)$ values calculated in approximation (3.3). This discrepancy has the principal character and is caused by the difference between the mechanisms of change of the wave number $b$ and, consequently, of the OP modulation period $P \sim b^{-1}$ in the approaches (3.3) [9] and [1-4]. In the model (3.3) the growth of modulation period $P=2 \pi / b$ with decreasing the temperature is described by taking into account the non-linear invariant $\left(-g \cdot \varphi^{2} \varphi^{2}\right)$ in TP, which leads to the following dependence of wave number on the temperature $T$

$$
b^{2}=\frac{\gamma}{2}+\frac{1}{8} g a^{2}
$$

where $a=a(T)$ is the OP amplitude [9].

In the approach in [1-4] the alteration of modulation period $P=4 K(k) / b(K(k)$ being the complete elliptic integral of the first order) is caused by the non-linear properties of distribution (3.4) and can take place also in the absence of the invariant $\left(-g \cdot \varphi^{2} \varphi^{2}\right)$. This property of distribution (3.4) enlarges the abilities of model (2.1) in the description of space-inhomogeneous states of the systems of different nature which can be characterized by the varying values of $\varphi^{2} \varphi^{\prime 2}$-interaction.

The OP dependence on the coordinate $x$ for three values of effective temperature $q$ is shown in Fig. 1. It obviously demonstrates the basic features of OP parametric evolution in the incommensurate phase with decreasing temperature, namely: a) the growth of OP amplitude, 
b) the increase of the modulation period, c) the rise

\begin{tabular}{||c|c|c|c||c|c|c||c|c|c||}
\hline $\mathrm{q}$ & $\Phi$ & $\Phi$ & $\Phi$ & $\varphi(0)$ & $\varphi(0)$ & $\varphi(0)$ & $\varphi^{\prime \prime}(0)$ & $\varphi^{\prime \prime}(0)$ & $\varphi^{\prime \prime}(0)$ \\
& $(3)$ & $(4)$ & $\mathrm{n}$ & $(3)$ & $(4)$ & $\mathrm{n}$ & $(3)$ & $(4)$ & $\mathrm{n}$ \\
\hline .2 & $-1.95 \mathrm{e}-4$ & $-1.96 \mathrm{e}-4$ & $-2.01 \mathrm{e}-4$ & .126 & .126 & .126 & -.060 & -.059 & -.058 \\
\hline 0 & $-5.24 \mathrm{e}-3$ & $-5.41 \mathrm{e}-3$ & $-5.40 \mathrm{e}-3$ & .297 & .298 & .299 & -.116 & -.097 & -.096 \\
\hline-.2 & $-1.88 \mathrm{e}-2$ & $-2.09 \mathrm{e}-2$ & $-2.11 \mathrm{e}-2$ & .437 & .457 & .460 & -.114 & -.031 & -.021 \\
\hline
\end{tabular}

Table. The results of numerical investigation of order parameter distributions in the incommensurate phase. Here $q$ stands for the effective temperature, $\Phi$ is the reduced thermodymanical potential, $\varphi(0), \varphi^{\prime \prime}(0)$ are initial conditions for the $S_{02}-$ set of initial conditions. The notation '(3)' stands for the 'model (3)', '(4)' means 'model(4)', 'n' denotes 'numerical'. The material parameters are equal to $g=-10, p=1$.

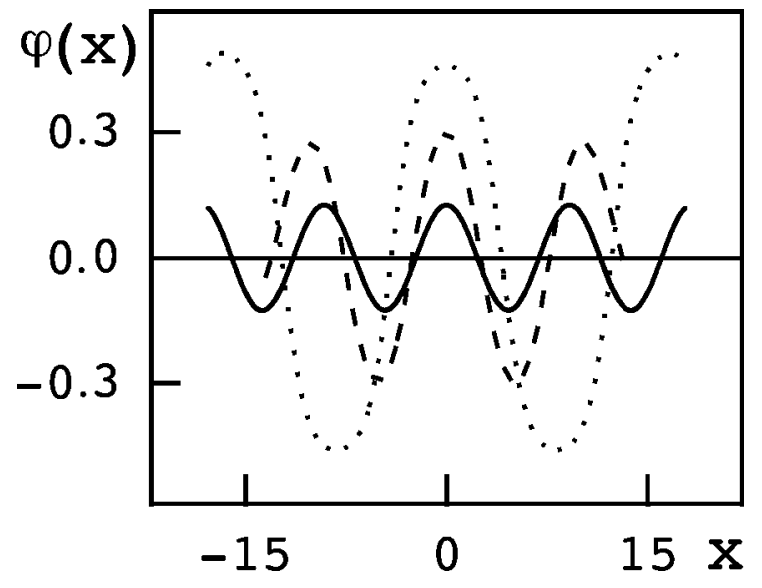

Fig. 1. The order parameter dependence on the coordinate $x$. The solid curve corresponds to the case when the effective temperature is equal to $q=0.2$, the dashed curve depicts the case $q=0.0$, the dotted curve is for $q=-0.2$. All calculations are carried out for $g=-10$.

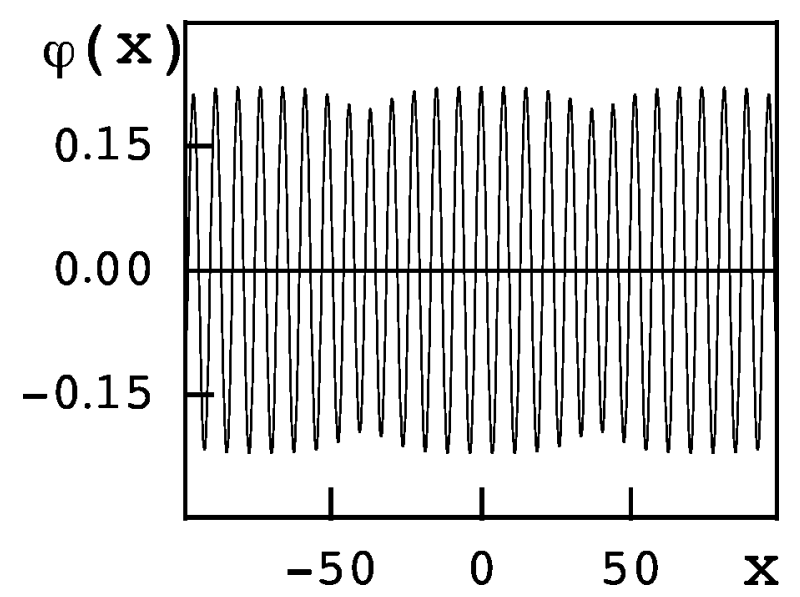

Fig. 2. The typical non-equilibrium distribution of order parameter $\varphi(x)$. The material parameters and initial conditions are equal respectively to $q=0.0, g=-10$. and $\varphi(0)=0.222, \varphi^{\prime \prime}(0)=-0.149, \varphi^{\prime}(0)=\varphi^{\prime \prime \prime}(0)=0$.

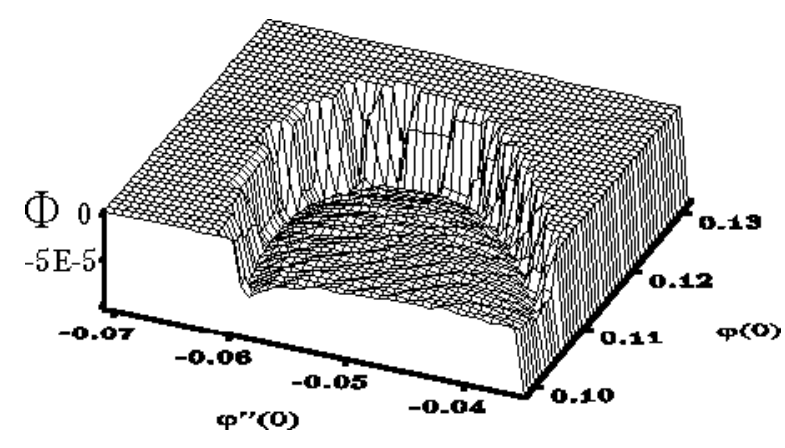

Fig. 3. The characteristic dependence of thermodynamical potential $\Phi$ on the initial conditions. The material parameters are equal to $q=0.2, g=-10$.

of contribution of higher harmonics (i.e. the tendency to transform into the domain walls lattice).

The characteristic feature of the investigated numerical distributions, regardless of their correspondence to the TP minima, is that, in general, they can be presented as the superposition of two oscillations with different amplitudes and different periods (Fig. 2). From the mathematical point of view such a behaviour is obvious. Really, the characteristic equation for the linearized variant of equation (2.2) is biquadratic. Hence, in general, the periodic solution of linearized variational equation (2.2) has the form of the sum of two harmonic oscillations with different wave numbers $b_{1}, b_{2}$ :

$$
\begin{aligned}
& \varphi(x)=a_{1} \cdot \cos \left[b_{1}\left(x+x_{01}\right)\right]+a_{2} \cdot \cos \left[b_{2}\left(x+x_{02}\right)\right] \\
& b_{1,2}=\frac{1}{2}[1 \pm \sqrt{1-4 q}]
\end{aligned}
$$

As it follows from (3.6), the relation $b_{1} / b_{2}$ continuously changes with the alteration of $q$ and, in general, is arbitrary.

The two-frequency structures also exist far from the point $q_{I}$ in the vicinity of which the linear approximation for equation (2.2) is correct. 
At the same time, the equilibrium OP distributions obtained in models (3.3) and (3.4) are single-wave nonharmonic oscillations which can be characterized by the only one wave number $b_{1}$. The Fourier series for the distribution (3.4) contains harmonics for which the relation

$$
b_{2}=(2 n+1) \cdot b_{1}, \quad n=0,1,2 \ldots
$$

is correct $\left(b_{1}\right.$ is the wave number of the main harmonic (3.3)). Only for the functions for which the relation (3.7) holds the TP value can be deeper than that one for the one-harmonic distribution (3.3) [14]. On the other hand, if the expression for the OP distribution contains an additional harmonic which does not satisfy relation (3.7) then the TP value increases. Hence, the solution which corresponds to the TP absolute minimum has the form of single non-linear wave but not of the superposition of two or more oscillations with arbitrary wave numbers $b_{1}, b_{2} \ldots$ However, even for the ideal crystal some factors always exist due to which a "mono-frequent" distribution is not realized and, in fact, the structures like the one plotted in Fig. 2 arises (as our calculations show, such states are always characterized by a higher TP value than the equilibrium distributions). Really, in experiments, the ordered state of system is usually obtained after the system hardening from the mono-phase disordered state. As a consequence, some metastable states can appear. These metastable states, as a rule, have the non-exponential law of relaxation $[11,15]$.

The considered additional oscillations (Fig. 2) take place against the background of space-periodic waves of the ground state. Such oscillations play the role of static perturbations of the equilibrium OP distribution and have the character of periodic structure of the nontopological dark solitons [16]. It should be noted, that the solution of Fig. 2 type are more numerically stable than the single-wave distributions which correspond to the TP minimum.

The typical TP dependence on the initial conditions is shown in Fig. 3. To simplify the analysis in Fig. 3 we set $\Phi=0$ for the solutions which give positive TP values.
As Fig. 3 shows, the region of TP minimum is not a single narrow "well" on the TP surface in the space of initial conditions. It has both the lines of sharp and smooth change of the TP value. Keeping in mind this fact we can suggest that if the system has been prepared in the inequilibrium state which is close to the equilibrium one then it would relax to the static state in two stages. On the first stage the system quite quickly comes running to some "valley" of minima. The second stage corresponds to the slow relaxation along the lines of a smooth change. Such a behaviour (the phenomena of "large river-bed") is rather an ordinary one for the non-linear relaxational equations [17]. It should be noted that the description of space-inhomogeneous states on the basis of model (2.1) weakly depends on the microscopic details of the considered system and has a unique character for the different condensed matters (ferroelectrics, magnetics, binary alloys and so on). Hence, the mentioned relaxational behaviour can be considered as yet another manifestation of the phase transition universality $[17,18]$. But the detailed discussion of this problem calls for the approaches which explicitly take into account the kinetic processes.

Therefore, the numerical investigation of the variational problem (2.1)-(2.2) shows that the approach in [1-4] quantitatively describes the OP distribution in the incommensurate phase, in particular, in the vicinity of "lock-in" transition point. The weak perturbation of equilibrium OP distribution has the form of periodic structures of the type of dark solitons on the background of space-periodic waves of the ground state. The relaxation to the equilibrium state is suggested to proceed in the two stages — "fast" and "slow".

\section{ACKNOWLEDGMENTS}

The research described in this publication was supported, in part, by the State Fund of Fundamental Researches of Ukraine under Grant No. 2.4/691. S. V. Berezovsky is also indebted to the Ministry of Science and Technology of Ukraine, scholarships 235/96.
[1] V. F. Klepikov and S. V. Berezovsky, Cond. Matt. Phys. (Lviv) iss. 8, 69 (1996).

[2] V. F. Klepikov, A. I. Olemskoi, S. V. Berezovsky, Metallofiz. Nov. Tekhnol. 19, 32 (1997).

[3] S. V. Berezovsky, V. F. Klepikov, V. Yu. Korda, Ukr. Fiz. Zh. 42, 889 (1997).

[4] S. V. Berezovsky, V. F. Klepikov, V. Yu. Korda, N. A. Shlyakhov, Int. J. Mod. Phys. B12, 365 (1998).

[5] J. C. Tolédano and P. Tolédano, The Landau Theory of Phase Transitions (World Scientific Publ. Co., Singapore, 1987).

[6] H. Z. Cummins, Phys. Rep. 185, 211 (1990).

[7] A. F. Jacobs, M. B. Walkers, Phys. Rev. B 21, 4132
(1980).

[8] H. Shiba, Y. Ishibashi, J. Phys. Soc. Jpn. 44, 1592 (1978).

[9] Y. Ishibashi and H. Shiba, J. Phys. Soc. Jpn. 45, 409 (1978).

[10] O. B. Arushanyan, S. F. Zaletkin, Chisliennoie rieshieniie obyknoviennykh diffierientsial'nykh uravnienii na fortranie (The numerical solving of the ordinary differential equations on FORTRAN) (Moscow State Univ. Publ., Moscow, 1990).

[11] A. I. Olemskoi, V. F. Klepikov, I. V. Koplyk, O. B. Krutko, A. V. Khomenko, Met. Phys. Adv. Tech. 16, 125 (1996). 
[12] A. D. Bruce, R. A. Cowley, J. Phys. C 11, 3609 (1978).

[13] V. F. Klepikov, Sov. Phys. - Low Temp. Phys. 17, 1166 (1991).

[14] A. D. Bruce, R. A. Cowley and A. F. Murrey, J. Phys. C 11, 3591 (1978).

[15] A. I. Olemskoi, I. V. Koplyk, Usp. Fiz. Nauk 165, 1105 (1995).

[16] A. M. Kosevich, A. S. Kovalev, Vhiedieniie v nieliniei- nuiu fizichieskuiu miekhaniku (The introduction in the non-linear physical mechanics) (Naukova Dumka, Kyiv, 1989).

[17] A. S. Zeltser, T. K. Soboleva, A. E. Filippov, Zh. Eksp. Teor. Fiz. 108, 356 (1995).

[18] A. I. Olemskoi, A. V. Khomenko, V. F. Klepikov, Ukr. Fiz. Zh. 41, 756 (1996). 